\title{
Los desafios epistemológicos de la educación científica para el desarrollo de la agricultura en Cuba
}

\section{The epistemological challenges of Science Education for the development of agriculture in Cuba}

\author{
Adolfo Ramos Lamar ${ }^{1}$ \\ https://orcid.org/0000-0003-1164-1172 \\ Eduardo Francisco Freyre Roach ${ }^{1}$ \\ https://orcid.org/0000-0002-2267-1564
}

\begin{abstract}
Resumen: Hoy en día el desarrollo de la agricultura es inconcebible sin la formación de científicos agropecuarios facultados para producir, aplicar, transferir y mejorar los conocimientos y tecnologías agropecuarios. Sin embargo, existe en el mundo un amplio consenso académico de que la agricultura sea sostenible, tanto en lo económico, como social y ambiental. Esto demanda un cambio de perspectiva epistemológica de la educación científica agropecuaria. El trabajo es de índole teórica y sistematiza y analiza informaciones y experiencias de trabajo docente y de investigación de los autores sobre el cambio epistemológico en la educación agropecuaria superior en Cuba, atendiendo a los cambios de políticas y como se concretan esos cambios en el trabajo investigativo y docente de los estudiantes y professores universitarios.
\end{abstract}

Palabras claves: Epistemología. Educación científica. Agricultura sostenible. Cuba.

\begin{abstract}
Currently the development of agriculture is inconceivable without the training of agricultural scientists empowered to produce, apply, transfer and improve agricultural knowledge and technologies. However, there is broad academic consensus in the world that agriculture is sustainable, economically, socially and environmentally. This demands a change in the epistemological perspective of agricultural science education. The paper is theoretical and unprecedented. It systematizes and analyzes relevant information, and experiences in teaching and research work by the authors on the epistemological change in higher agricultural education in Cuba, taking into account the changes in policies and how these changes have been concretized in the research and teaching involving students and university professors.
\end{abstract}

Keywords: Epistemology. Science education. Sustainable agriculture. Cuba.

\footnotetext{
${ }^{1}$ Fundação Universidade Regional de Blumenau (FURB), Programa de Pós-graduação em Educação (PPGE), Blumenau, SC, Brasil. E-mail: jemabra@furb.br
} 


\section{Introducción}

Para Bunge (2009) "[...] la ciencia y la técnica son el motor de la civilización moderna". De eso no cabe la menor duda. Importantes aspectos de nuestras vidas serían hoy inconcebibles sin la producción y aplicación de conocimientos científicos, por ejemplo, la salud pública, la economía, la alimentación, la educación, las comunicaciones, la internet, el disfrute del tiempo libre, etc. No por casualidad lo que un país invierte y avanza en ciencia y tecnología constituye indicador de su prosperidad y desarrollo, y comparación con otros países.

La educación científica resulta un factor indispensable para potenciar su desarrollo. Sin embargo, la satisfacción de esta expectativa demanda reeplantear determinados presupuestos filosóficos y epistemológicos, referidos, sobre todo, a la naturaleza del saber científico, sus condicionamientos e impactos, así como en su relación con otros saberes.

Es oportuno no perder de vista que: "A lo largo de la historia de la humanidad, las ideas acerca de la ciencia han ido evolucionado en el tiempo, apareciendo diferentes modelos o visiones que en cierta forma han intentado integrar las formas de pensamiento de la época marcando etapas en su desarrollo" (ASENCIO CABOT, 2014, p. 551).

Un cambio en la idea de ciencia, investigación científica y en los criterios cientificidad de la comunidad científica lleva a cambios en la educación científica, en sus propósitos de formación y estrategias docentes. Ciertamente la educación científica reproduce la concepción de la ciencia dominante en el "paradigma" de la comunidad científica: en los libros de texto o manuales, así como en el trabajo de investigación experimental que los estudiantes realizan, en correspondencia con las definiciones y aplicaciones de los conceptos, teorías, y métodos que aprenden. En este sentido, y como se infiere de $A$ estrutura das revoluções científicas, de Thomas Kuhn (KUHN, 1978), la educación científica constituye un factor de conservación de ese paradigma, pero también resultaría un escenario donde se detectan anomalías, y por tanto, la crisis que daría paso a una revolución científica. Y este suceso, puede venir incluso de estudiantes que no están muy fuertemente adoctrinados en el paradigma que les están enseñando. Producir, aplicar, enseñar y aprender conocimientos científicos presupone también una determinada imagen de lo qué la ciencia es, de cómo hacer ciencia, con qué métodos, qué es o no susceptible de investigación científica. Tal imaginario del proceder científico no es estático, sino cambiante.

Aquí nos interesa la educación científica agropecuaria, la formación de profesionales agropecuarios de nivel superior, que se se facultan para aprender, producir, aplicar, mejorar, transferir, e, inclusive, enseñar conocimientos científicos y tecnologías para el desarrollo agropecuario. Hoy en día el desarrollo de la agricultura es inconcebible sin este perfil profesional.

Partimos también del consenso académico global de que la agricultura ha de ser sostenible, tanto en lo económico, como social y ambiental. La crisis ambiental lo demanda. Como es archiconocido, a partir de lo que se conoce como Revolución Verde, se han globalizados practicas agrícolas modernas como los monocultivos, el uso de fertilizantes y plaguicidas químicos, la selección y propagación de variedades genéticas, así como el uso de combustible fosiles, y maquinarias, etc. Empero, el uso inadecuado de estos procedimientos ha sido responsable de perturbaciones ecológicas como la degradación, erosión y contaminación de los suelos, las aguas, el aire, la deforestación, y la reducción de la biodiversidad. Hoy en día se plantea el imperativo del desarrollo sostenible de la agricultura o de la agricultura sostenible. Es decir, una agricultura que satisfaga las necesidades de productos y servicios agropecuarios para las 
generaciones presentes y futuras, sin comprometer el medio ambiente, y el acceso equitativo a los recursos naturales y los bienes agropecuarios.

De ahí entonces, que las universidades en el mundo entero tienen ante si el reto de introducir modificaciones en el currículo de las carreras agropecuarias en correspondencia con esta perspectiva o racionalidad que requiere el desarrollo sostenible de la agricultura. En Brasil se vienen aportando algunas experiencias en cuanto a la implementación de un currículo de educación científica agropecuaria de pregrado y postgrado que precisamente contribuya a formar científicos agropecuarios capacitados profesionalmente de gestionar el desarrollo sostenible de la agricultura (JACOB et al., 2016). Como se verá en este trabajo la peculiaridad de la experiencia cubana en tal sentido consiste en el alcance no solo local, sino también nacional de tal modificación curricular de la educación científica agropecuaria. El enrumbamiento de la educación científica agropecuaria en esta dirección implica ya no sólo la introducción de nuevos conocimientos científico, sino también un cambio en el modo de entender qué es la ciencia, las relaciones entre las ciencias, con las tecnologías, y el saber campesino, así sus condicionamientos e impactos sociales, y la responsabilidad social y ética en el trabajo científico. Es decir, cuestiones de Filosofía de la Ciencia, Filosofía de la Tecnología, y Epistemológia entran en escena a la hora de pensar el desarrollo de las ciencias agropecuarias y la educación científica agropecuaria en nuestros países.

Dada esta situación esbozada, en este trabajo se plantea el problema científico siguiente: ¿En que medida el enrumbamiento de la educación científica agropecuaria en Cuba en correspondencia con el enfoque de la agricultura sostenible y implica ya no solo la introducción de nuevos conocimientos científicos. El objetivo de este trabajo consiste en abordar este cambio de paradigma epistemológico que está experimentando la educación científica agropecuaria en Cuba en las últimas décadas, dada la adopción oficial de la agricultura sostenible como directriz de la política agropecuaria.

La investigación realizada es de carácter teórico. Se obtienen los datos mediante la exploración de la bibliografía disponible y la sistematización de experiencias docentes y de investigación de los autores que durante dos décadas trabajaron como profesores en las carreras de ciencias agropecuarias, educación, ciencias sociales y otras carreras en Cuba.

El primer epígrafe versa sobre el estatuto epistémico de la investigación y la educación en ciencias agropecuarias. Se trata de una investigación de índole teórica sobre un tema académico que viene suscitando debates y controversias en el mundo. Tal identidad epistemológica suele ser no reconocida por quienes traen a colación y enfatizan la intencionalidad técnica, aplicada o practica de las ciencias agropecuarias.. La defensa del estatuto epistémico de las ciencias agropecuarias constituye el fundament teorico general de esta reflexion teorica que se expone en este trabajo sobre el cambio epistemológico de la educación agropecuaria superior en Cuba. A continuación se explica como ese cambio epistemológico es consecuencia de las reformas que el gobierno cubano introdujo en la político agraria, y otras políticas para enfrentar la crisis económica en 1990. Los epígrafes siguientes presentan evidencias empíricas de como ese cambio epistemológico se refleja en el trabajo científico de los estudiantes y profesores vinculados a programas y proyectos de desarrollo sostenible, local y endogeno. Cerramos el trabajo mostrando como el cambio epistemológico que ha comenzado a gestarse en la educación superior agropecuaria en Cuba encuentra concreción en los contenidos docentes de la agenda curricular que precisamente abordan la problemática epistemológica. 


\section{E1 status epistemológico autónomo de las ciencias agropecuarias y la educación científica agropecuaria}

Lo primero que se impone dejar bien aquí en claro es el status epistemológico de las ciencias agropecuarias y la educación científica agropecuaria.

Por lo general, y partir de la era moderna, cuando se habla de las llamadas ciencias básicas, fundamentales o clásicas, no suele cuestionarse su status de ciencias. Pero ese cuestionamiento se ha hecho con respecto a las denominadas ciencias sociales, las ciencias aplicadas y, las ciencias técnicas, como las agrícolas o agropecuarias. Tal demarcación presupone siempre un paradigma determinado de lo que es la ciencia, donde se toman ciencias modelos como la Matemática, la Física, la Química, y la Biología. Hoy en día la negación del status de ciencia basada en esa extrapolación conceptual, resulta un anacronismo epistemológico.

En primer lugar, sería negligente desdeñar que la agricultura es un ámbito específico que requiere no solo de la aplicación de ciencias conocidas como fundamentales o básicas, o de transferencias de tecnologías, sino también de un conocimiento específico del ámbito agropecuario en sí mismo (FREYRE ROACH; RAMOS LAMAR, 1994). Es decir, que el status de ciencias de las ciencias agropecuarias en conjunto y selectivamente (Agronomía, Fitotecnia, Medicina Veterinaria, Mecanización Agropecuaria, etc.) se fundamenta en esta ontología de lo agrícola o lo agropecuario como realidad objetiva susceptible de investigación científica. Actualmente se viene hablando de Agricultural ontology ya no sólo en el sentido de Ontología como rama filosófica de lo que existe (del ser o la realidad), sino como un modelo para clasificar, construir y desarrollar conceptos y bases de datos (asistido por computadora), y sus relaciones, de interés para el manejo de los sistemas agrícolas (LAUSER et al., 2006).

En segundo lugar, considérese que no es lo mismo, analizar un contexto agrícola (parcela, finca, empresa o región, etc.) en sus diferentes elementos (el suelo, el agua, el clima, las especies, etc.), que integrar todos los elementos, es decir, considerar que la unidad básica sería el agroecosistema, su estabilidad, resiliencia y sostenibilidad. Eso significa que las ciencias agrícolas resultan un conocimiento científico peculiar, sobre todo por el requisito de la integración de conocimientos, los conceptos transversales a cada una de las ciencias agropecuarias, y el propósito tecnológico e ingenieril del manejo eficiente de los sistemas agrícolas.

En tercer lugar, como ocurre en todas las ciencias, en las agropecuarias existen percepciones diferentes de como realizar ese manejo o gestión agrícola de los recursos naturales. Por ejemplo, un asunto es un sistema de manejo de una finca que considere al suelo agrícola como un mero soporte físico-químico para la nutrición de las plantas y los animales, o como receptor de residuos agrícolas. Otro es que al suelo se le entienda como un organismo vivo, por lo cual las estrategia agronómica son diferentes. En el primer caso se trata de nutrir las plantas, y en el segundo caso nutrir el suelo. Si antes el énfasis se coloca en el monocultivo o el ganado, ahora el objeto fundamental es el agroecosistema, la biodiversidad, y la integración de cultivos y ganadería. Es decir, la perspectiva de hacer ciencia agrícola es diferente. Los sistemas de monocultivos por lo general se basan en el primer enfoque, los sistemas agroecológicos están promoviendo el segundo enfoque.

Lo que hoy se conoce como Agroecología ya no se reduce a la aplicación de principios ecológicos en el entendimiento y manejo sustentable de los agroecosistemas, sino que abarca todo un rango amplio de campos de conocimientos, y, destaquemos aquí el reeplanteamiento 
de la epistemología de las ciencias agrícolas, que viene abriendose paso en la educación científica agropecuaria.

La integración de conocimientos científicos en un curriculo de formación científica agropecuarias "es un medio idoneo para aprender la ciencia" (THOMPSON; WARNICK 2007, p. 2, nuestra traducción), y agregaríamos también para la reflexión epistemológica sobre la ciencia. No es difícil suponer que los profesores de ciencias agropecuarias pasan a las generaciones futuras de científicos agropecuarios no sólo los logros científicos, sino también un determinado entendimiento de lo qué es la ciencia en general y la ciencia específica que se trata.

En este sentido, hay profesores que ponen el énfasis en que los estudiantes conozcan los logros científicos y sepan aplicarlos a los casos que presuponen una determinada teoría o paradigma. A otros les interesa que los estudiantes sepan producir conocimientos científicos, y por lo tanto, se concibe una docencia que puntualiza en cómo esos conocimientos fueron logrados, cuáles conocimientos existían anteriormente, cuáles son las dificultades sin resolver, y que investigaciones habría que hacerse en el futuro. Es decir, una cosa es la ciencia ya hecha, y otra la que está por hacer.

Hay profesores de ciencias que ponen más interés en lo que se supone que deban enseñarle a los estudiantes, pero otros se concentran en el aprendizaje, es decir, en lo que el estudiante aprende. Si en el primer caso se corre el riesgo de la recepción pasiva de conocimientos, en el segundo se propicia la oportunidad de una docencia diferenciada, es decir, no homogénea.

Por último, cabe reiterar aquí que la educación científica no es mera transmisión o reproducción del modo de hacer y entender la ciencia, sino también de su desarrollo.

\section{El giro de la política agraria cubana a partir de los 1990 y sus consecuencias para la educación científica agraria}

Como se conoce, la revolución que se dio en la Química en el XX, tuvo gran repercusión en la agricultura y, específicamente aparecieron nuevos medicamentos veterinarios y fitosanitarios, los fertilizantes y plaguicidas agroquímicos, sintéticos o inorgánicos. El avance en la Biología cambió los métodos de selección genética, y con ello, la obtención de variedades y especies híbridas con gran potencial de crecimiento, producción, y rendimiento, pero que son demandantes de altos insumos industriales y recursos. La Física impulsaría sobre todo a la mecanización agropecuaria, empero, exigiría el uso intensivo de combustibles fósiles.

Todo estos avances científicos y productivos correspondientes se integraron en lo que se denominó la Revolución Verde. Los apologetas de este enfoque, empezando por su promotor pionero, Norman E. Borlaug suelen alegar que gracias a ese enfoque fue posible aumentar los rendimientos agrícolas por unidad de hectáreas y resolver el problema del hambre en todo el mundo. Y que las críticas ecologistas o ambientalistas al modelo de agricultura de Revolución Verde resultan incompatibles con el progreso de las ciencias.

En Cuba, durante los 1970 y los 1980, el enfoque de Revolución Verde constituía la directriz oficial científica, tecnológica, y política de la agricultura. Y por tal motivo hasta 1990 la agricultura cubana venía dependiendo de las inyecciones de fertilizantes, plaguicidas, medicinas veterinarias, petróleo, y maquinarias. Estos recursos y la asesoría científica y técnica requerida fue posible gracias a los acuerdos bilaterales de importación y exportación con la 
Unión de Repúblicas Socialistas Soviéticas (URSS) y otros países miembros del sistema de cooperación socialista de Europa del Este, el entonces llamado Consejo de Ayuda Mutua Económica (CAME). En este pilar descansaron, principalmente, los planes de desarrollo económico. Esta es la circunstancia histórica que consolidó la imposición y reproducción del modelo de desarrollo agropecuario de Revolución Verde, demandante de grandes cantidades de insumos y recursos industriales y exógenos, y orientado a la producción agropecuaria en sistemas de monocultivos y ganadería intensiva. Este modelo de desarrollo agropecuario implica la introducción de avances de las ciencias modernas.

Pues bien, en 1989 ocurre el derrumbe del Muro de Berlín, y se disuelve la URSS. Tras este colapso del socialismo, la administración republicana del presidente Georg Bush recrudece el bloqueo económico impuesto a Cuba desde 1961. Lógicamente, la agricultura cubana y el sistema alimentario son embestidos por una recia crisis. Por la falta de insumos agropecuarios, los planes agropecuarios, quedan sin realización. Debe tomarse en cuenta que ya antes de la crisis se habían detectado y reconocido oficial y popularmente ciertos problemas en la producción agropecuaria: la erosión de los suelos; la insuficiencia de incentivos para vivir y trabajar en el sector agropecuario; la no correspondencia entre la disponibilidad y el uso de insumos, la productividad y los rendimientos. Esta impronta vuelca a Cuba en dirección a lo que oficialmente refrendó como "Periodo Especial en tiempos de paz" o "Economía de Guerra". Entonces viene la necesidad de un replanteamiento tanto de la política agrícola, como de la política en ciencia e innovación tecnológica, en la política educacional, en la política, que obviamente repercute en la epistemología de educación científica agropecuaria. Se plantea entonces la problemática de qué ciencia agropecuaria debería enseñarse y ser aprendida por el futuro profesional científico agropecuario, en sintonía con las reformas de la política agrícola (ÁLVAREZ FIGUEROA, 1997).

Uno de esos cambios transcendentales es el redimensionamiento de la tenencia de la tierra, el mercado agropecuario y la gestión agropecuaria. Merece destaque que en 1993 el Consejo de Estado emite el Decreto Ley no. 142 sobre las Unidades de Producción Cooperativa (UBPC) que estipula la entrega en usufructo gratuito de las tierras estatales a trabajadores de las empresas agropecuarias estatales. Este proceso de descentralización y cooperativización de la tenencia y la gestión agrícola, va mucho más lejos a partir del Decreto No. 259 de 2008 sobre la entrega de tierras ociosas a todo aquel que esté dispuesto a trabajarla y producir alimentos. Este proceso, digamos de "recampesinizar", no solo tiene importancia sociologíca, económica y cultural, sino también científica y epistemológica. El éxodo rural comienza a disminuir, pero también, emerge un escenario demográfico y sociológico idóneo para la valorización y rescate del saber tradicional de los campesinos y su relación con el saber científico.

Estamos, efectivamente ante una problemática epistemológica puntual. Pues ya el científico agropecuario no puede seguir produciendo conocimientos al margen del conocimiento campesino tradicional. La nueva generación de conocimientos científicos agropecuarios han de emerger de ese diálogo, que ya ha de comenzar en el proceso de la formación de científicos para la agricultura. En este contexto la imagen epistemológica de la ciencia como conocimiento superior no es concebible a expensa de minimizar la potencialidad del saber campesino. No pensar así sería otro anacronismo epistemológico.

Otro cambio de la política de repercusión epistemológica en la educación científica agropecuaria es que entre las fechas de estos decretos y en correspondencia con la Agenda 21, 
se dicta en 1997 la Ley No. 81 de Medio Ambiente, que, entre otros aspectos estipula el fomento de la Agricultura Sostenible, es decir de la producción agropecuaria económicamente viable y socialmente aceptable, en armonía con el medio ambiente. El mencionado Decreto No. 259 estipula que los nuevos tenedores de tierras las usen con vista a producir alimentos, pero de forma sostenible. No es difícil darse cuenta que en esta coyuntura disminuye la hegemonía de la Revolución Verde y se le otorga relevancia a la Agricultura Sostenible, y, por su puesto, a su epistemología. Un giro en tal sentido es que, como ya planteamos, que el agroecosistema se convierte en el objeto de interés científico fundamental, pero hay que agregar que ya no es posible pensar en la ciencia centrada en la superespecialización productiva y científica. La unidad del agroecosistema no admite la atomización o reduccionismo de los elementos que lo conforman. Se requiere, claro está de una visión holística o multidimensional de la realidad agricola y el conocimiento científico agropecuario.

En este contexto aparece la perspectiva docente de una educación científica agropecuaria cuyos contenidos y actividades docentes giran alrededor del objeto agroecosistema y de la producción de conocimiento acerca de las interacciones de esos elementos diversos, de su estabilidad, y resilencia, es decir, la capacidad de restablecer su equilibrio oportunamente.

\section{El trabajo científico de los estudiantes: de la parcela experimental a la experimentación en condiciones reales de producción}

¿Cómo es que este cambio epistemológico se refleja en el currículo de la formación de científicos agropecuarios?

En primer lugar, una vez que el objeto de trabajo científico da relevancia a los agroecosistemas, se desprende que el estudio, la investigación, el trabajo y la labor extensionista de los estudiantes se desplazan a las condiciones reales de producción, y, por supuesto, tributan a la misma. El Sistema de Ciencia, Tecnología e Innovación Tecnológica (SCIT) vigente en Cuba favorece este cambio epistemológico de la educación científica en los cursos agropecuarios. Esta política estipula que la ciencia, la tecnología y la innovación tecnológica constituyen herramientas fundamentales para el desarrollo económico y social del país. Y, en tal sentido, se aboga por la introducción sistemática y acelerada de los resultados de la ciencia, la innovación y la tecnología en la producción y los servicios.

En esta política de SCIT, se reitera aquí de forma enfática el compromiso de impulsar la agricultura sostenible, la creatividad de los colectivos laborales de base y su participación en la solución de los problemas tecnológicos. Sin embargo, dadas las nuevas condiciones, ya no resulta viable la hegemonía o generalización del anterior sistema de empuje tecnológico (technology-push), a través de paquetes tecnológicos, basados en el uso intensivo de insumos, y respondiendo, no al sistema de market pull, sino a los objetivos estatales (DÍAZ et al., 2007). La política de descentralización administrativa pone al orden del día la visión de que el desarrollo tecnológico debe satisfacer con más creces las demandas locales de los productores del sector cooperativo emergente y en expansión. Es por eso que se requiere con urgencia y sistematizidad "el fomento de un enfoque participativo "de abajo hacia arriba" (NUÑEZ JOVER; MONTALVO ARRIETE, 2015, p. 13), que proveerá mayor espacio para valorizar, conservar, $\mathrm{y}$ difundir el conocimiento tradicional.

Finalmente, como parte del SCIT, merece consideración que en Cuba desde finales de la década de los 90 funciona un sistema cienciométrico de evaluación (resultados, premios, 
patentes, publicaciones, citas, etc.) de los resultados de la ciencia, la tecnología y la innovación tecnológica (GONZÁLEZ GUITIÁN; MOLINA PIÑEIRO, 2008). Tal registro no se reduce a los resultados de las instituciones que producen conocimientos científicos, sino que contemplan también las innovaciones de los trabajadores y campesinos en las unidades de producción agropecuaria. Esto evidentemente constituye un dispositivo institucional para la valorización social del conocimiento local, obrero y campesino, el diálogo de este saber y el saber científico, y la difusión nacional de los resultados. De igual forma mecanismos como el Fórum de la Ciencia y la Tecnología (FCT) y la Asociación de Innovadores y Racionalizadores (ANIR) dan relevancia a los resultados de profesores, investigadores, estudiantes, extensionistas, trabajadores, campesinos, y la población en general, que contribuyen al desarrollo tanto nacional como local.

"En Cuba, como en otros países de la América Latina, gran parte de las capacidades científicas y tecnológicas están concentradas en las universidades. No es simple, sin embargo, conectar esas capacidades con las necesidades de la vida cotidiana de las personas." (NUÑEZ JOVER et al., 2015, p. 230). Hoy en día, como hemos dicho, hay espacio para la producción de conocimientos a todos los niveles. Los estudiantes de ciencias agropecuarias tienen incentivos y espacios para hacer valer su ingenio científico, y respondiendo a las necesidades del sector productivo. El contexto experimental deja de reducirse al aula o el laboratorio. Las entidades productivas pasan a ser los principales centros donde la educación científica agropecuaria estudiantil comienza a desarrollarse.

Esta dirección que toma el SCIT se refleja en la dinámica de los planes de estudios A, B, C, y D (ÁLVAREZ DE ZAYAS, 1996). Gran parte del trabajo metodológico del claustro de profesores de ciencias agropecuarias se pone en función de satisfacer tanto la demanda de la integración de conocimientos y técnicas, como el trabajo práctico en las entidades productivas mismas.

Ese trabajo metodológico entraña los desafíos epistemológicos que hemos ido esbozando. Siendo el agroecosistema la unidad básica de análisis de la investigación agropecuaria, significa que el trabajo científico se hace más complejo. El estudiante ha de capacitarse en la investigación interdisciplinar y cooperativa, en el diseño y manejo de policultivos. Si antes el contenido del programa de estudio daba relevancia a los monocultivos y la especialización, ahora se trata del manejo científico de agroecosistemas complejos donde las variables no son controlables como ocurre en el laboratorio experimental. Obviamente, dadas las actuales transformaciones el objetivo profesional viene vertiginosamente desplazándose hacia al sector campesino: la Cooperativa de Pequeños Campesinos (CPA), las Unidades Básicas de Producción Cooperativa (UBPC), las Cooperativas de Créditos y Servicios (CCS), y los campesinos individuales.

Seguido a esto, vivimos el comienzo de cambios en el sistema de extensionismo agrario universitario. Ya se torna obsoleto el sistema de extensión agraria de índole linear, ofertista, transferencista, vertical, y burocrática, que imperaba en la universidad, a fin de cuentas implicó la estigmatización y subvaloración del saber tradicional de los campesinos (LÓPEZ, 2005). Como se dijo el SCIT envía señales en otra dirección.

No es difícil constatar que en este contexto de los cambios de programas de estudios en las carreras agropecuarias, va ganando terreno, la formación de un científico agropecuario con perfil amplio y no estrecho, debido a que los sistemas agropecuarios o de investigación que 
solo atienden a un aspecto (un recurso: suelo, semilla, maquinaria, fertilizante), vienen siendo desplazados por el concepto de agroecosistemas. Esto resulta un cambio epistemológico transcendental. Podríamos resumir ese cambio epistemológico que viene abriéndose paso en los planes de estudios de ciencias agropecuarias, en los siguientes aspectos:

- La unidad básica de trabajo científico y experiental son los agroecosistemas en condiciones reales de producción, por lo que la experimentación científica no se reduce al laboratorio.

- Los resultados de la investigación científica de los estudiantes resuelven problemas concretos de la producción agropecuaria en el ámbito local, lo cual requiere de actualización ya no solo de los conocimientos científicos, sino también del cambio de factores a esa dimensión.

- La educación científica tiende más a la mutidisciplinariedad que a la especialización estrecha, es, una formación científica más integral y conectada con la práctica productiva real.

- El estudiante agropecuaria aprende a valorizar el saber de los campesinos y productores, y se forma en la capacidad de asesorar la producción y aprender de los campesinos.

\section{Experiencias concretas del cambio epistemológico a través de los programas y proyectos de desarrollo sostenible, local y endógeno de la agricultura}

Como un ejemplo de que el enfoque de la agricultura sostenible en suelo universitario viene propiciando la valorización del saber tradicional y el diálogo de saberes, se puede mencionar la participación en el movimiento de campesino a campesino (CAC), que, auspiciado por la Asociación de Técnicos Agrícolas y Forestales (ANAP), provee empoderamiento de los campesinos y promueve el diálogo de conocimientos entre los mismos y de estos con los profesores, investigadores y extensionistas de los cursos agropecuarios. Dicha metodología de CAC se concibió como el sistema de métodos, procedimientos y técnicas que facilitan desencadenar procesos de intercambio y aprendizaje entre los campesinos(as) y sus familias, así como entre dirigentes, técnicos, investigadores y otros actores relacionados (MACHÍN SOSA et al., 2012).

En este contexto se observa "[...] a campesinos e investigadores conversando e investigando de igual a igual, y obteniendo muy buenos resultados en el conocimiento y la producción" (CUBA..., 2016, p. 4). Y esto resultó en una especie de revolución epistemológica, porque el enfoque agroecológico de la agricultura sostenible requiere de saberes locales, creatividad, principios, protagonismo campesino, el rescate de prácticas populares y/o ancestrales, su capacidad de innovación local (ROSSET, 2016).

El CAC fortalece los vínculos y convenios de colaboración entre las cooperativas de campesinos, los centros de investigación y las universidades a todo lo largo y ancho del país. Con el Periodo Especial, Cuba entra en una etapa "donde la inteligencia y creatividad de campesinos, obreros, técnicos y profesionales del sector se puso a prueba, en aras de la sostenibilidad agrícola" (MACHÍN SOSA et al., 2016, p. 26). La entrada del enfoque CAC en las universidades está acercando los centros de producción y formación agropecuaria a los entornos del saber campesino. Obviamente las líneas de investigación agraria universitaria tratan de responder a este desafío.

Tributando a proyectos como este de CAC y otros, las universidades cubanas en los últimos tiempos están promocionando: 
la recuperación y difusión de los saberes campesinos... debiendo ser un agente activo en la legitimación y aprendizaje de conocimiento campesino, lo que implica aceptar que el conocimiento científico es una forma de saber, exige humildad y apertura para entablar un diálogo de saberes" (RIZOMUSTELIER; VUELTA-LORENZO; LORENZO-GARCÍA, 2017, p. 8).

Con la relevancia que en la pasada década se le otorgó al desarrollo local, se constituyó en el Ministerio de Educación Superior (MES) en el 2006 el Programa de Gestión Universitaria del Conocimiento y la Innovación para el Desarrollo (GUCID). Entonces “[...] apareció así en la agenda universitaria cubana el tema del desarrollo local y su articulación con las actividades de ciencia, tecnología e innovación” (NUÑEZ JOVER et al., 2015, p. 233).

A este programa tributa con éxito el Programa de Innovación Agropecuaria Local (PIAL) del Instituto Nacional de Ciencias Agrícolas (INCA), perteneciente al Complejo Científico Docente Universidad Agraria de la Habana (UNAH). En este escenario se realiza el proyecto de Fitomejoramiento Participativo (FP) con el protagonismo de los productores y la colaboración de profesores, investigadores, extensionistas y estudiantes de las universidades. Para el Dr. Humberto Díaz Labrada, el FP consiste en un sistema en que se ofrecen variedades de laboratorio y locales, siendo los campesinos los protagonistas del proceso de su selección, experimentación y adopción, "atendiendo a las características específicas de sus suelos, medioambiente y cultura” (MARTÍN GONZÁLEZ, 2010, p. 1). Un aspecto importante del PIAL que tributa al rescate del saber campesino y el diálogo de saberes es que propicia el empoderamiento de los agricultores y su desempeño como consultores de los Ministerios de la Agricultura y el Azúcar de Cuba.

En la misma UNAH cabe destacar los proyectos del Centro de Desarrollo Agrario y Rural (CEDAR), inicialmente impulsados por la Dra Nilda Pérez Consuegra y el Dr. Rafael Ojeda Suárez, quien fuera su Director. El CEDAR viene poniendo énfasis en el engranaje entre las actividades docentes y de investigación de los estudiantes y profesores y la producción agraria local. Los Talleres de Sistematización de Experiencias Locales vienen conectando sinérgicamente los profesionales y campesinos, así como los saberes académicos y tradicionales (OJEDA SUÁREZ, 2006), lo cual transciende el campo de la agricultura, y abarca otras esferas que aseguran el bienestar de la población en sus localidades. Una proyección importante de este centro es que coloca en manos de los productores locales herramientas de informática que les permiten contar con información de gran utilidad para el manejo de los agroecosistemas.

\section{El papel de los contenidos de Epistemología en la educación científica en la enseñanza superior agropecuaria}

El redimensionamiento de los contenidos de epistemología a través de los cursos de filosofía es un factor clave en este cambio epistemológico de la educación agropecuaria. Como parte de las transformaciones de los planes y programas de estudios agropecuarios en los cursos de enseñanza superior agropecuaria, consideración especial merece la aprobación ministerial de la asignatura Problemas Sociales de la Ciencias y la Tecnología (PSCT) y con ella surgió un marco de trabajo importante para discutir las concepciones de ciencia y tecnología, su naturaleza, alcance cognoscitivo e impactos sociales y ambientales. 
En ambas perspectivas epistemológicas ya mencionadas ya veníamos trabajando incluso antes de la crisis de los 1990, cuando en el currículo de la formación científica agropecuaria comenzamos a incorporar contenidos docentes relacionados. Por ejemplo, los estudiantes reflexionaban y debatían enfoques nuevos de la ciencia en los trabajos de autores clásicos tanto del mundo socialista como John D. Bernal, B. Hessen, S. Mikulinski, etc. Muchos profesores cubanos que imparten cursos de epistemología en las ciencias agropecuarias que fueron formados en Cuba, así como en la Unión Soviética y Alemanía, conocían estos enfoques, y también de otras tradiciones de pensamientos y autores como L. Laudan, A. Musgrave, A. Koyré, B. Hessen, L. Fleck. G. Bachelard, P. K. Feyerabend, M. Polanyi, K. R. Popper, T. S. Kuhn y I. Lakatos, E. Morin, M. Bunge, J. D. García Bacca, C. U. Moulines y L. Olive, entre otros. De hecho, incluso, investigadores de la ciência de la União Soviética no desconocían los trabajos epistemológicos sobre la ciência realizados por autores del Occidente (STEPANOVNA, 1974).

Aunque no se deben menospreciar las diferencias de enfoques, los autores mencionados confluyen en cuanto desafían la imagen positivista clásica de la ciencia. Tal concepto positivista de la ciencia, articulada en los trabajos de Comte, Spencer, Mill, Durkheim, Mach, Avenarius y el Circulo de Viena, cerraba el camino para el reconocimiento de las ciencias agropecuarias como ciencias e implicaba también el estigma, la desvalorización, y hasta la persecución y exclusión del conocimiento no científico tradicional y sus productores, los obreros, campesinos, y personas corrientes. Merece acotarse lo siguiente. El Marxismo pauta la reflexión epistemologica de las ciencias en Cuba y en varios puntos Marx y los positivistas confluyen:

[...] creen en la superioridad intrínseca del método científico. Aceptan por igual el papel de la ciencia como guía en el camino hacia el progreso. Marx es también un firme creyente en la sensatez de pretender 'adueñarse' de la naturaleza, como una manera de explotar las riquezas naturales en beneficio de los hombres, pero también como un medio de arrebatar al mundo natural su carácter sagrado impuesto por 'las tradiciones supersticiosas de los primitivos' y recientemente recuperado por el Romanticismo. Marx, como todos los positivistas, ve en el mito una forma básica de la falsedad, de lo erróneo y fácticamente equivocado. La ciencia, desde luego, librará a la humanidad de la superstición y de los mitos. Engels, por su parte, detalla el camino evolucionista que ha seguido el trabajo, como actividad plenamente humana, en su 'marcha hacía el progreso' (los pulgares oponibles, según esta visión, nos habrían hecho 'progresar' sobre los simios). (ROARO, 2012, p. 65).

Como muy bien se conoce en la Unión Soviética y en Cuba se impuso una versión de la filosofía de Marx y Engels donde finalmente sobresalen estas afinidades. Por lo tanto, la introducción de contenidos basados en las críticas al positivismo representaban también una critica a ese Marxismo, y de ahí, al replanteamiento de la identidad epistemologica en las ciencias agropecuarias en la red nacional de formación de profesionales agropecuarios.

Por otro lado, al claustro de profesores de los cursos agropecuarios no le era ajena la problemática ambiental de la agricultura. Ya en los años 80, profesores de agronomía comenzaban a prestar atención a los trabajos de ecología, agroecología, manejo integrado de plagas, 
control biológico de plagas, los abonos orgánicos, y la integración agricultura-ganadería, etc. Los profesores de filosofía y epistemología comenzamos a introducir contenidos de la teoría de la biosfera de Vladimir Vernadsky (1863-1945) y de la teoría de la noosfera de Pierre Teilhard de Chardin (1881-1955).

Nuestras investigaciones y estudios sobre cuestiones filosóficas y sociales de las ciencias agropecuarias de los años 90 corren en esta perspectiva epistemológica (FREYRE ROACH; RAMOS LAMAR, 1994). Más allá de esto, los contenidos de PSCT comenzaron a exigirse como requisito de ascenso académico, no sólo en el nivel pregrado, sino también de postgrado, y tanto para profesores, como investigadores y directivos. "Hasta la fecha, no hay otro curso que tenga esa posición privilegiada dentro del ámbito de la enseñanza superior agropecuaria en Cuba” (RAMOS LAMAR; FREYRE ROACH, 2018, p. 434).

Un contenido epistemológico importante de nuestros cursos es el cuestionamiento de la imagen positivista de la ciencia y la tecnología. Con ello se descalifica la idea positivista de la ciencia como conocimiento absolutamente benefactor y neutral. Esto es muy importante tenerlo en cuenta en la educación científica agropecuaria, pues dada esta convicción positivista de la ciencia, se impuso también la concepción de que todo resultado científico por el mero hecho de ser debe ser aplicado a toda costa. En Cuba ha venido ocurriendo que las instituciones productoras de conocimientos científicos para la agricultura, por un lado, gozan de condiciones favorables para la aplicación de sus resultados en la producción agropecuaria, pero, por otro lado, muchos resultados no se aplican dado a que no responden a las necesidades y al contexto de esa producción, y no falta la resistencia por parte de los productores. Hoy en día esta problemática se viene debatiendo en el contexto de la discusión sobre los proyectos del gobierno de introducir los Organismos Genéticamente Modificados. En Cuba hay un movimiento nacional de profesores y campesinos, y de organizaciones no gubernamentales que viene alertando al gobierno de los riesgos económicos y ambientales de esta tecnologia (ALTIERI, 2012; FUNES-MONZOTE; FREYRE ROACH, 2009). Quienes defienden esta tecnología en Cuba suelen argumentar que esta tecnología es un progreso de la ciencia que seria descabellado oponerse a su difusión y generalización.

En este sentido debe ser de comprensión de los estudiantes las repercusiones ambientales y sociales adversas de las tecnologías agropecuarias de Revolución Verde. La desvalorización del conocimiento de los campesinos ha sido uno de los factores que han provocado problemas en los recursos naturales, sino también en la composición demográfica del campo.

Otro contenido es el referente a la relación entre ciencia y tecnología. Una vez que se entiende que esta relación no es linear, entonces se comprende también, que el asunto en educación agropecuaria no es la mera aplicación, adaptación o transferencia de un conocimiento ya elaborado, sino que el conocimiento desde su concepción hasta su aplicación tiene que armonizar ambos intereses.

Otro grupo de contenidos epistemológico que debemos destacar aquí es el abandono de la idea convencional de la ruptura o el dualismo epistemológico entre las ciencias agropecuarias y las ciencias sociales. Sobre este asunto epistemológico hay gran consenso entre el claustro de profesores que labora en la educación científica agropecuaria en Cuba. Pero más allá, debe tomarse en cuenta que ya desde finales de los 1990 los institutos universitarios especializados en determinado grupo de ciencias, comienzan a fusionarse en universidades y filiares universitarias locales, donde interactúan los estudiantes de ciencias agropecuarias con 
las de otras carreras como la Economía, la Ingeniería Industrial, la Contabilidad, los Estudios Socioculturales, las Ciencias de la Computación, y el Derecho.

\section{Consideraciones finales}

Hay hoy en Cuba un cambio epistemológico en la educación superior agropecuaria y las universidades estan tratando de fortalecer su participación en el desarrollo local. En Cuba la crisis económica de los 90 y el redimensionamiento de la política agraria y los planes y programas de estudios en los cursos agrarios vienen propiciando la valorización, el rescate y la relevancia del saber científico y tecnológico moderno, pero también del saber campesino, y del diálogo de saberes.

Enfoques del desarrollo agrario sostenible en Cuba propician esa sinergia de saberes académicos universitarios y tradicionales campesinos. Por cuanto han surgidos nuevos productores con poca experiencia agrícola constituye un reto para las universidades y los campesinos la capacitación de esos productores. Existe gran consenso en que esto requiere de la educación superior agropecuaria la realización de diagnósticos sobre las necesidade capacitación, satisfacer las demandas locales de conocimiento, y facilitar la eficacia productiva y social en las unidades emergentes de producción (VALLEJO ZAMORA et al., 2016). Una de las vías ha sido sin duda alguna la medida que estimula la participación de los estudiantes en los proyectos de investigación y extensión de las facultades, departamentos, cursos y profesores.

Merece destacarse aquí aquellos proyectos con participación protagónica de campesinos. Esto se fomenta en gran medida por el grado de educación de los campesinos cubanos y de los nuevos productores. El enfoque de la agricultura sostenible exhibe hoy logros y oportunidades, pero también dificultades y desafíos en la implementación de este enfoque. La educación científica agropecuaria viene operando una perspectiva epistemologica distinta a la convencional, dictada por el enfoque de Revolución Verde, y las políticas de desarrollo de la agricultura y la educacion. Como cambiaron los tiempos, las circunstancias y las políticas, entonces emerge el replanteamiento que potencia el desarrollo sostenible y los cambios epistemologicos, que se reflejan en los planes y programas de estudios agropecuarios, en la investigación, la extensión, la enseñanza de la filosofía y los problemas sociales de la ciencia y la tecnologia.

Como en el Brasil, en Cuba, y en el mundo en general:

O campo universitário é um lugar de lutas para determinar as condições e os critérios de pertencimento e de hierarquia legítimas. Em outras palavras, é um local caracterizado por disputas entre diferentes grupos para definir quais são as regras de conduta que devem ser seguidas ou, por exemplo, as metodologías de pesquisa que são aceitas para a produção dos conhecimentos que o campo oferece. (BORSATTO; CARMO, 2013, p. 7).

Lo es el contexto de la investigación científica agropecuaria y la educación científica agropecuaria, donde actualmente se enfrentan el enfoque de la agricultura convencional o de Revolución Verde y el enfoque del desarrollo sostenible de la agricultura y la Agroecología. 
Como hemos mostrado en nuestro país esta polémica se viene desarrollando teniendo como marco de fondo las dificultades y transformaciones de la política agrícola. Pero el cambio epistemológico en curso enfrenta barreras y obstáculos como la apreciación quizás ya no romántica, sino nostálgica de que lo ideal es perfeccionar el modelo de Revolución Verde, y que la agricultura sostenible tiene importancia solo coyuntural, a falta de recursos, y dada la situación del bloqueo económico que Estados Unidos ejerce sobre Cuba.

De todas maneras, la necesidad de la sostenibilidad de la agricultura no es una cuestión encerrada en esta condición. Por un lado, la erosión de los suelos y la deforestación, la redución de la diversidad genética, así como la hegemonía de las cooperativas de fincas diversificadas en Cuba hace pensar en la imposibilidad de volver al uso intensivo de insumos industriales y los sistemas de monocultivos.

Por otro lado, este cambio ofrece posibilidades y oportunidades inéditas para la educación científica agropecuaria en Cuba. En fin, no es menos cierto que el cambio epistemológico que viene ocurriendo en las ciencias y la educación agraria en Cuba emerge de la coyuntura histórica de crisis económica de 1990. Cuando se absolutiza este carácter táctico temporal, se corre el riesgo de que cuando se den las condiciones de recursos se vuelva al imaginario epistemologico anterior. De ahí que en trabajos anteriores hemos indicado la recepción romántica y nostálgica de quienes en Cuba apuestan a volver a la perspectiva de la Revolución Verde.

Sin embargo, consideramos que el cambio rumbo a la agricultura sostenible tiene un alcance que va más allá de la coyuntura en que emergió. Primero, por la citada emergencia ambiental, alertada por profesionales de la agricultura y de las ciencias sociales mucho antes de la crisis. Pero en segundo lugar porque el debate epistemológico dentro y fuera de Cuba ha propiciado un cambio de la concepción de la ciencia en sintonía precisamente con la perspectiva de la sostenibilidad de la agricultura.

En Brasil se vienen emprendiendo acciones locales, comunitarias y gubernamentales con vistas a la introducción de la perspectiva orgánica, agroecológica y sustentable en la producción agrícola, pero, sobre todo, a pequeña escala y con vistas a satisfacer la demanda de productos orgánicos en el mercado interno (CAMPANHOLA; VALARINI, 2001). Se reportan en tal sentido el incremento de experiencias comunitarias mayormente a escala pequeña sobre todo en las regiones del sur del Brasil, por ejemplo en cooperativas de la Associação de Agricultura Orgânica do Paraná (AOPA) (HESPANHOL, 2008). Lógicamente a la par se registran iniciativas con vistas a la formación ambiental y agroecologica en la formación de profesionales de nivel superior capaces de contribuir al desarrollo sustentable de la agricultura en Brasil (MARCOMIN; SILVA, 2009). De hecho, vienen aumentando los cursos y facultades donde las modificaciones curriculares abren espacio para contenidos docentes que contribuyan a esa formación (BALLA; MASSUKADO; PIMENTEL, 2014; VIEGAS; CABRAL, 2015).

Ahora bien, autores brasileños oportunamente llaman la atención de que la potenciación de una agricultura ecológica no debe asociarse solo con la impronta medioambiental en su estrecho sentido conservacionista o proteccionista del medio ambiente, sino que deben considerarse otras dimensiones, la social y la económica, cultural, política y ética (CAPORAL; COSTABEBER, 2001).

Independientemente de que el sistema agrícola de Brasil y Cuba difieren en diversos puntos, la experiencia cubana proporciona un marco de referencia de interés para quienes están trabajando en Brasil con vistas al desarrollo agrícola sustentable. Primero, el caso cubano 
en tal sentido, se caracteriza por el fuerte apoyo gubernamental, no gubernamental, y comunitario, popular y local. Segundo, que las instituciones de Educación y los sectores públicos y privados (sector campesino cooperativo) cooperan y emprenden acciones conjuntas. Tercero, las iniciativas agroecológicas en educación cuentan con facilidades para su difusión a todo lo largo y ancho del territorio (FUNES AGUIAR; FREYRE; BLANCO, 2016).

\section{Referencias}

ÁLVAREZ DE ZAYAS, C. M. El diseño curricular en la educación superior cubana. Pedagogía Universitaria, La Habana, v. 1, n. 1, p. 36-50, 1996. Recuperado de: http:/ cvi. mes.edu.cu/peduniv/index.php/peduniv/article/view/103/102. Visitado en: 10 out. 2019 ÁLVAREZ FIGUEROA, O. E1 sistema educativo cubano en los noventa. La Habana: Centro de Investigaciones de Economía Mundial, 1997.

ALTIERI, M. A. La paradoja de la agricultura cubana. [2012]. Recuperado de: http:/ / www.revista.ecaminos.cu/articles/article/la-paradoja-de-la-agricultura-cubana-reflexiones-a/. Visitado en: 11 abr. 2015.

ASENCIO CABOT, E. Una aproximación a la concepción de ciencia en la contemporaneidad desde la perspectiva de la educación científica. Ciência $\boldsymbol{\&}$

Educação, Bauru, v. 20, n. 3, p. 549-560, 2014. DOI: https://doi.org/10.1590/151673132014000300003.

BALLA, J. V. Q.; MASSUKADO, L. M.; PIMENTEL, V. C. Panorama dos cursos de agroecologia no Brasil. Revista Brasileira de Agroecologia, Dois Vizinhos, PR, v. 9, n. 2, p. 3-14, 2014. Recuperado de: http://revistas.aba-agroecologia.org.br/index.php/ rbagroecologia/article/view/15589.Visitado en: 26 mar. 2019.

BORSATTO, R.; CARMO, M. S. A agroecologia como um campo científico. Revista Brasileira de Agroecologia, Dois Vizinhos, PR, v. 8, n. 2, p. 4-13. 2013. Recuperado de: http://revistas.aba-agroecologia.org.br/index.php/rbagroecologia/article/view/12890. Visitado en: 26 mar. 2019.

BUNGE, M. Somos víctimas de una mala filosofía política: entrevista a Mario Bunge [por Alberto Montero]. Newropeans Magazine, Nijmegen,16 jul. 2009.

CAMPANHOLA, C; VALARINI, P. J. A agricultura orgânica e seu potencial para o pequeno agricultor. Cadernos de Ciência e Tecnologia, Brasília, v. 18, n. 3, p. 69-101, 2001.

CAPORAL, F. R.; COSTABEBER J. A. Agroecologia e desenvolvimento rural sustentável: perspectivas para uma nova extensão rural. In: ETGES, V. E. (org.). Desenvolvimento rural: potencialidades em questão. Santa Cruz do Sul: Edusc, 2001. p. 19-52.

CUBA: su nueva revolución campesina. Biodiversidad: sustento y culturas, La Habana, n. 90, p. 3-4, 2016. Recuperado de: https://www.grain.org/es/article/5599. Visitado en: 10 oct. 2019. 
Lamar, A. R.; Roach, E. F. F.

DÍAZ, J. A. et al. J. Reflexiones acerca de la transferencia de tecnologías en el sector ganadero: el sistema de extensionismo del Instituto de Ciencia Animal (SEICA) en Cuba como estudio de caso. Revista Cubana de Ciencia Agrícola, La Habana, v. 41, n. 2, p. 157-163, 2007.

FREYRE ROACH, E. F; RAMOS LAMAR, A. Ciencias agrarias y sociedad. In: NÚÑEZ JOVER, J. Problemas sociales de las ciencias y las tecnologías. Ciudad Habana: Editorial Félix Varela, 1994. p. 15-28.

FUNES AGUILAR, F.; FREYRE, E.; BLANCO, F. Capacitación agroecológica. In: FUNES AGUILAR, F; VÁZQUEZ MORENO, L. L. (ed.). Avances de la agroecología en Cuba. La Habana: EE. Indio Hatuey, 2016. p. 449-468.

FUNES-MONZOTE, F. R.; FREYRE ROACH, E. F. Transgénicos: quien gana, quien pierde. La Habana: Centro Félix Varela de la Habana: Editorial Acuario, 2009.

GONZÁLEZ GUITIÁN, M. V.; MOLINA PIÑEIRO, M. La evaluación de la ciencia y la tecnología: revisión de sus indicadores. Revista Cubana de los Profesionales de la Información y la Comunicación en Salud, La Habana, v. 18, n. 6, p. 1-19, 2008.

JACOB, L. B.; ALMEIDA JR., A. R.; AZEVEDO, M. A. R.; SPAROVEK, G. A agroecologia nos cursos de engenharia agronômica: para além de desafios e dilemas curriculares.

Avaliação, Campinas, v. 21, n. 1, p. 173-198, 2016. DOI: https://doi.org/10.1590/S141440772016000100009.

HESPANHOL, R. A. M. Perspectivas da agricultura sustentável no Brasil. Confins: revista franco-brasileira de geografía, Marseille, n. 2, 2008. DOI: https://doi.org/10.4000/ confins. 2353 .

KUHN, T. S. A estrutura das revoluções científicas. 2. ed. São Paulo: Perspectiva, 1978.

LAUSER, B.; SINI, M.; LIANG, A.; KEIZER, J.; KATZ, S. From AGROVOC to the agricultural ontology service / concept server: an OWL model for creating ontologies in the agricultural domain. In: PROCEEDINGS of the 2006 international conference on Dublin core and metadata applications: metadata for knowledge and learning. [S.l.]: Dublin Core Metadata Initiative, 2006. p. 76-88.

LÓPEZ, T. Organización y estructura del sistema de extensión agraria (sea) en Cuba.

Revista Computadorizada de Producción Porcina, La Habana, v. 12, n, 1, p. 22-26, 2005.

MACHÍN SOSA, B.; JAIME, A.; LOZANO, D.; ROSSET, P. (org). Revolução

agroecológica: o movimento de camponês a camponês na ANAP em Cuba. São Paulo:

Expressão Popular, 2012.

MACHÍN SOSA, B.; ROQUE JAIME, A. M.; ÁVILA LOZANO, D. R.; ROSSET, P.

M. Transformaciones de la agricultura cubana". Biodiversidad: sustento y culturas, La Habana, n. 90, p. 21-26, 2016. Recuperado de: https://www.grain.org/es/category/534biodiversidad-90-2016-4. Visitado en: 10 oct. 2019. 
MARCOMIN, F. E.; SILVA, A. D. A sustentabilidade no ensino superior brasileiro: alguns elementos a partir da prática de educação ambiental na universidade. Revista Contrapontos, Itajaí, v. 9, n. 2, p. 104-117, 2009.

MARTÍN GONZÁLEZ, M. Los campesinos tienen que protagonizar las ciencias agropecuarias. Juventud Rebelde, La Habana, 29 mayo 2010. Recuperado de: http:/ /www. juventudrebelde.cu/cuba/2010-05-29/los-campesinos-tienen-que-protagonizar-las-cienciasagropecuarias. Visitado en: 8 ago. 2010.

NUÑEZ JOVER, J; MONTALVO ARRIETE, L F. La política de ciencia, tecnología e innovación en Cuba y el papel de las universidades. Revista Cubana de Educación Superior, La Habana, v. 34, n. 1, p. 29-43, abr. 2015. Recuperado de: http://scielo.sld.cu/ scielo.php?script $=$ sci_arttext\&pid $=$ S0257-43142015000100003\&lng $=$ es\&nrm $=$ iso. Visitado en: 22 mayo 2018.

NUÑEZ JOVER J.; ARMAS MARRERO, I.; ALCÁZAR QUIÑONES, A.; FIGUEROA ALONSO, G. Educación superior, innovación y desarrollo local: Experiencias en Cuba. Congreso Universidad, La Habana, v. 4, n. 3, p. 228-248, 2015. Recuperado de: http:/ / revista.congresouniversidad.cu/index.php/rcu/article/view/715/677. Visitado en: 10 oct. 2019.

OJEDA SUAREZ, R. Gestión del conocimiento en el desarrollo local. In: LA NUEVA universidad cubana y su contribución a la universalización del conocimiento. La Habana: Editorial Félix Varela, 2006. p. 21-30.

RAMOS LAMAR, A.; FREYRE ROACH, E. El giro epistemológico en la Universidad Agraria de La Habana (UNAH), Cuba. Revista Internacional de Educação Superior, Campinas, v. 4, n. 2, p. 425-437, 2018. DOI: https://doi.org/10.20396/riesup.v4i2.8650752. RIZO-MUSTELIER, M.; VUELTA-LORENZO, D. R.; LORENZO-GARCÍA, A. M. Agricultura, desarrollo sostenible, medio ambiente, saber campesino y universidad. Ciencia en su PC, Santiago de Cuba, n. 2, p. 106-120, 2017. Recuperado de: http:/ /www.redalyc. org/articulo.oa?id=181351615008. Visitado en: 22 de mayo 2018.

ROARO, J. Marxismo y positivismo. Disputatio: philosophical research bulletin, Salamanca, España, v. 1, n. 1, p. 63-76, 2012. Recuperado de: https://gredos.usal.es/ handle/10366/124003. Visitado en: 20 mayo 2019.

ROSSET, P. Las recetas no funcionan, lo que se propone son principios. Biodiversidad: sustento y culturas, La Habana, n. 90, p. 5-10, 2016

STEPANOVNA, N. El postpositivismo y la nueva metafísica. Revista Problemas Filosóficos, Moscu, n. 1, 1974. (En russo).

THOMPSON, G. W.; WARNICK, B. K. Integrating science into the agricultural education curriculum: do science and agriculture teachers agree? Journal of Agricultural Education, West Lafayette, v. 48, n. 3, p. 1-12, 2007. 
VALLEJO ZAMORA, Y.; PÉREZ CASTRO, T.; POZO NÚÑEZ, E. M.; AROZARENA DAZA, N.; LÓPEZ LABRADA, A. La capacitación agraria desde la visión del agricultor, en el municipio Boyeros, La Habana, Cuba. Cultivos Tropicales, La Habana, v. 37, n. 2, p. 149-154, 2016.

VIEGAS, S. F. S.; CABRAL, E. R. Práticas de sustentabilidade em instituições de ensino superior: evidências de mudanças na gestão organizacional. Revista GUAL, Florianópolis, v. 8, n. 1, p. 236-259, 2015. 